\title{
ATOMISTIC MODELING OF LASER-RELATED PHENOMENA
}

\author{
Luis A. Marqués, María Aboy, Pedro López, Iván Santos, Lourdes Pelaz \\ Departamento de Electricidad y Electrónica, Universidad de Valladolid, Escuela Técnica \\ Superior de Ingenieros de Telecomunicación, 47011 Valladolid, SPAIN \\ Giuseppe Fisicaro \\ Consiglio Nazionale delle Ricerche, Istituto per la Microelettronica e Microsistemi (CNR- \\ IMM), Z.I. VIII Strada 5, I-95121 Catania, ITALY
}

\section{INTRODUCTION}

Although experimental tests are still essential for the design and optimization of new technologies in the semiconductor industry, modeling and simulation are becoming fundamental to reduce development times and costs. Nowadays atomistic modeling, along with more traditional continuum-based tools, are being used for processing simulation (Pelaz et al. 2009). Modeling is particularly important in order to study the effects of laser irradiation on semiconductor properties in both melted and solid phases (Lombardo et al. 2017), as laser processing is gaining relevance within the electronic industry. Many issues are still poorly understood, even for the widely investigated case of laser thermal annealing (LTA) of ionimplanted Si. For instance, the evolution of common dopant atoms in the melting phase, or the amount of dopant activation promoted by laser irradiation in solid regions (Monakhov et al. 2005; Ong et al. 2006; Fisicaro et al. 2013). Due to the intrinsic features of laser annealing treatments, i.e., localized irradiation with a space- and time-dependent (on the nanosecond scale) thermal fields which lead to far-from-equilibrium conditions, experimental analysis can reveal only specimen post-irradiation characteristics. In order to understand how the laserirradiated system evolves and reaches its final state, a theoretical work based on full process simulation is required.

Traditional models for laser processing simulation were based on continuum techniques. However, they prove to be insufficient at the nanoscale. Atomistic simulation methods, in turn, are handy for studying detailed interactions, both at the electronic and atomic levels, including out-of-equilibrium situations as those present in laser processing. However, the extension of atomistic simulation approaches which are valid for conventional annealing is not straightforward for laser processing conditions. On the one hand, energy transfer to the lattice comes from the excitation of the electronic system, which now has to be carefully modeled (especially in ultra-fast laser annealing) while it was completely ignored in conventional processing simulations. On the other hand, the characteristics of the thermal profiles induced by the laser are very demanding for numerical simulations, as they evolve very rapidly in time with substrate zones close to or above the melting point next to regions at room temperature. Additionally, atomistic parameters (diffusivities, bonding energies, pre-factors, etc.), which have been calibrated in conventional annealing conditions, have to be revised in order to be applied to laser annealing simulation as entropy effects may become relevant.

In this chapter we will review the main techniques for atomistic simulation to be applied for laser modeling, from the more fundamental ab initio and tight binding, to the less detailed Monte Carlo methods that allow reaching macroscopic size and time scales. In section 2 we will introduce the general features of these techniques, to then consider more specifically in section 3 the particular models routinely used for laser annealing simulation in each case. In section 4 we will present some recent results on atomistic modeling of laser-related phenomena in $\mathrm{Si}$, in particular melting and regrowth processes, defect and dopant kinetics, dopant 
segregation, and the anomalous formation of extended defects. Finally, in section 5 we will address some conclusions and future perspectives about the atomistic modeling of laser processing.

\section{ATOMISTIC SIMULATION TECHNIQUES}

In this section we will review the main techniques for atomistic simulation, and the need of using such techniques in a multi-scale scheme in order to reach time and space scales typical of real materials and processes.

\subsection{Ab initio}

$\mathrm{Ab}$ initio methods allow to determine fundamental properties of materials based on the laws of quantum mechanics. The underlying core is the resolution of the many-body Schrödinger equation for systems containing from tens to thousands of atoms and their corresponding electrons. Given the position of a set of atom nuclei $R$ and their electrons $r$, the many-body Schrödinger equation of the system is:

$$
H \Psi(R, r)=E \Psi(R, r) \quad \text { Eq. } 1
$$

where $\Psi(R, r)$ is the wavefunction of the system (atom nuclei and electrons), $H$ is its Hamiltonian, and $E$ the total energy of the system. The computational complexity grows exponentially with the number of atoms, and so several approaches have arisen to be able to solve it. Non-relativistic Born-Oppenheimer approximation assumes that electrons move much faster than nuclei, as they are thousands of times lighter. Consequently, the electronic and nuclei motion are decoupled. By means of this ansatz, and together with the adiabatic approximation (nuclei move on the potential-energy surface of the electronic ground state), the electronic system can be studied separately through this time independent Schrödinger equation:

$$
-\frac{\hbar^{2}}{2 m_{e}} \nabla^{2} \Psi_{e}(r ; R)+V(r ; R) \Psi_{e}(r ; R)=E_{e} \Psi_{e}(r ; R)
$$

being $\hbar=h / 2 \pi$ where $h$ is Planck's constant, $m_{e}$ the mass of the electron, $\Psi_{e}(r ; R)$ the wave function of the whole electronic system (with atom nuclei at positions $R$ ) once the electron and nuclei movement has been decoupled, and $V(r ; R)$ is the potential affecting the electron movement (that includes electron-nuclei and electron-electron interactions).

A well defined theoretical framework is the wavefunction-based approaches. The simplest one is the Hartree-Fock method, which produces reasonable results for many properties, by using the variational theorem to obtain an approximate solution. However, it neglects electron correlation in electronic structure calculations, which implies each electron accounts the rest of them as a mean-field. Another drawback is the high computational effort: calculations for a system composed of $N$ atoms scale as $N^{4}$ (Leach 2001). A complementary approach to HartreeFock is the Density Functional Theory (DFT), based on the Hohenberg-Kohn theorem (Hohenberg \& Kohn 1964), which states that the ground state electronic energy is uniquely determined by the electron density. Despite several approaches of the correct energy functional have to be made, DFT calculations are feasible for realistic models to accurately predict material properties.

$\mathrm{Ab}$ initio can be considered as the more fundamental atomistic technique since it gives excellent insight into the underlying physics of systems with no free parameters. Nevertheless, the high 
computational cost of ab initio limits the accessible system sizes and simulation times. Most of ab initio studies use simulation cells of about 200 - 300 atoms. Larger simulation cells are not commonly considered due to the computational overhead, although high performance computing facilities can be used for treating larger systems. The time scale accessible to ab initio molecular dynamics is typically of the order of several picoseconds. Although diffusion paths can be studied with ab initio molecular dynamics, this time scale is, in general, not enough for observing complete diffusion events, and consequently static methods such as the Nudget Elastic Band Method (Jónsson, Mills \& Jacobsen 1998) are commonly employed.

The ab initio technique is best suited to provide essential information on defects and dopants, to be subsequently used in front-end process models. For example, it has been employed to study the energetics of Si self-interstitials (Is) and vacancies (Vs) (Clark \& Ackland 1997; Mercer et al. 1998; ; Cargnoni, Gatti, \& Colombo 1998; Al-Mushadani \& Needs 2003) in order to find their most stable configuration and to obtain an atomistic interpretation of self-diffusion in Si (Blöchl et al. 1993); as well as to analyze the formation of Si self-I (Kohyama \& Takeda 1999; Coomer et al. 2001; Du et al. 2007) and V (Makhov \& Lewis 2004) clusters. The diffusion of dopants (Nichols, Van de Walle \& Pantelides 1989; Ramamoorthy \& Pantelides 1996; Zhu et al. 1996; Windl et al. 1999; Sadigh et al. 1999), and the formation and dissolution of dopantdefect complexes (Liu, Windl \& Masquelier 2000; Lenosky et al. 2000) have also been analyzed using ab initio techniques, providing information about which are the mobile species, and their formation and migration energies. Furthermore, ab initio simulations also enable studying the influence of charge states of dopant-defect complexes on the diffusion process (Windl et al. 1999).

\subsection{Tight binding}

As in the case of ab initio methods, the starting point of the tight binding (TB) method is the Schrödinger equation describing the system under study. After applying the Born-Oppenheimer approximation, the many particle problem is reduced to the problem of just one particle (electron) moving in an effective field due to the other electrons and the ions. In the case of an extended system such a crystal, Bloch (Bloch 1928) provided a formal procedure for developing the crystalline one-electron wavefunction on the basis of the atomic orbitals, which constitutes the so-called Linear Combination of Atomic Orbitals, Bloch, or just TB method. Unlike the parameter-free ab initio technique, an adequate parametrization of the Hamiltonian matrix elements is necessary to describe the system under study. This fact makes TB less computationally expensive than ab initio, but also less transferable. An extensive review of the TB method can be found in Colombo (2005).

The lower computational cost of TB enables accessing to longer simulation times and larger systems with respect to ab initio. Typical cells contain about several thousand atoms, and it is possible to simulate their dynamics during times of the order of hundreds of picoseconds. Nevertheless, TB can be linked to temperature accelerated dynamics schemes for easily accessing the nanosecond time scale (Cogoni et al. 2005). Consequently, diffusion paths can be obtained directly from the simulation without making any initial guess or resorting to static techniques. The formation and migration mechanisms of native point defects in Si (Tang et al. 1997; Munro \& Wales 1999; Jääskeläinen, Colombo \& Nieminen 2001; Colombo 2002), the recombination of Si self-Is and Vs (Tang et al. 1997; Zawadzki, Luo \& Clancy 2001), the formation energies of V and Si self-I clusters (Wang, Chan \& Ho 1991; Song et al. 1993; Arai, Takeda \& Kohyama 1997; Richie et al. 2004; Cogoni et al. 2005) and the levels they introduce in the gap (Song et al. 1993) are examples of different studies performed using TB simulation 